\title{
The Area of the Medial Parallelogram of a Tetrahedron
}

\author{
David N. Yetter \\ Department of Mathematics \\ Kansas State University \\ Manhattan, KS 66506
}

\begin{abstract}
The purpose of this note is to present a simple formula for the area of the medial parallelogram of a tetrahedron in terms of the lengths of the six edges, together with its derivation. It is not claimed that this formula is original, though diligent searches of standard sources of mensuration formulae, and inquiries to mathematicians in a variety of fields suggest that it is not generally known, and may be new. The author would be grateful for any references to previously published instances of this formula.

Despite the very classical nature of the problem it solves, there is some serious contemporary interest arising from recently proposed simplicial models for quantum gravity (cf. [1], [2]), in which such a formula is needed to approach the problem of length operators.

Consider a tetrahedron with edge-lengths as in Figure 1. Fix a pair of nonincident edges, say those of lengths $d$ and $e$. It is then easy to see that the midpoints of the remaining four edges lie in a plane parallel to both of the chosen edges, and equidistant from the planes containing each chosen edge and parallel to both, and that they form the vertices of a parallelogram in this plane.
\end{abstract}

Definition 1 Given a pair of non-incident edges in a tetrahedron, the medial parallelogram determined by the pair is the parallelogram whose vertices are the mid-points of the remaining four edges.

Our main result is then

Theorem 2 The area of the medial parallelogram determined by the edges of lengths $d$ and $e$ in the tetrahedron of Figure 1 is

$$
\frac{1}{8} \sqrt{4 d^{2} e^{2}-\left(b^{2}+f^{2}-a^{2}-c^{2}\right)^{2}}
$$

Proof: The key is to set the tetrahedron in a three dimensional coordinate system in a particularly convenient way. We do this by placing the vertex incident to the edges of lengths $a, b$, and $e$ on the positive $z$-axis, and the remaining vertices in the $x y$-plane, with the edge of length $d$ parallel to the $y$-axis. The vertices then have coordinates of the form $(0,0, z),(x, y, 0),(x, y+$ $d, 0)$, and $(\xi, v, 0)$. (See Figure 2.)

\footnotetext{
${ }^{1}$ Supported by NSF Grant \# DMS-9504423
} 


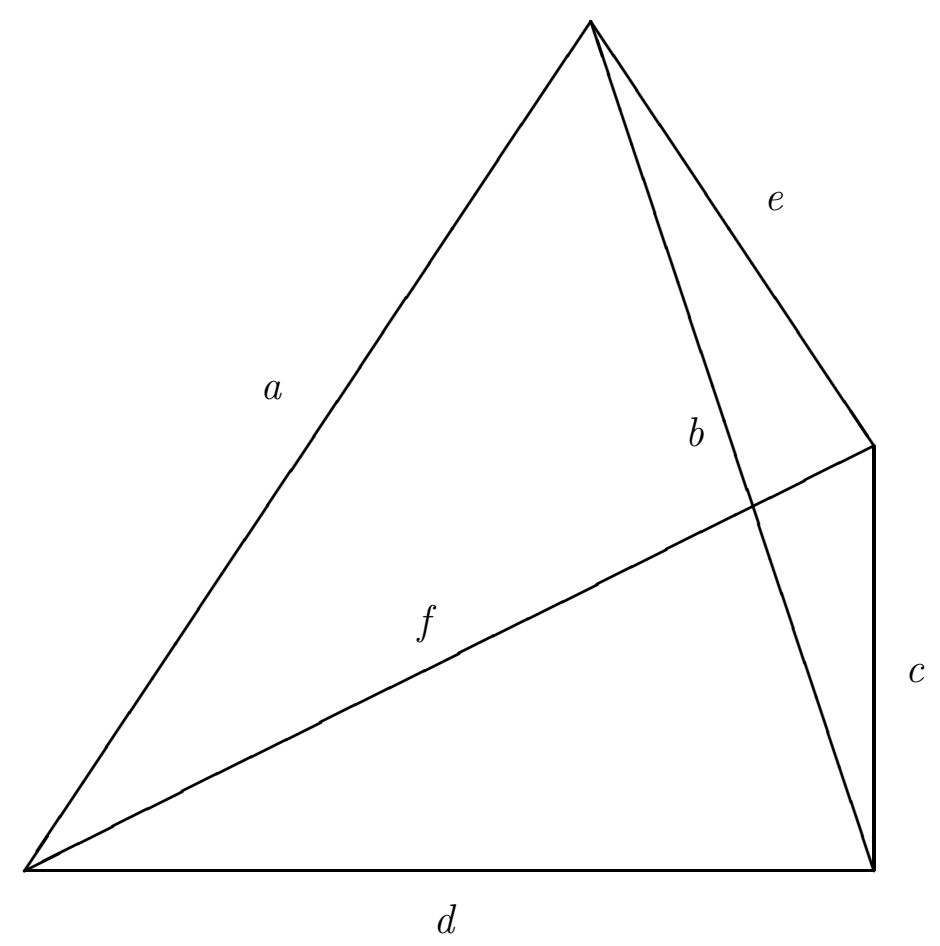

Figure 1: A generic tetrahedron 


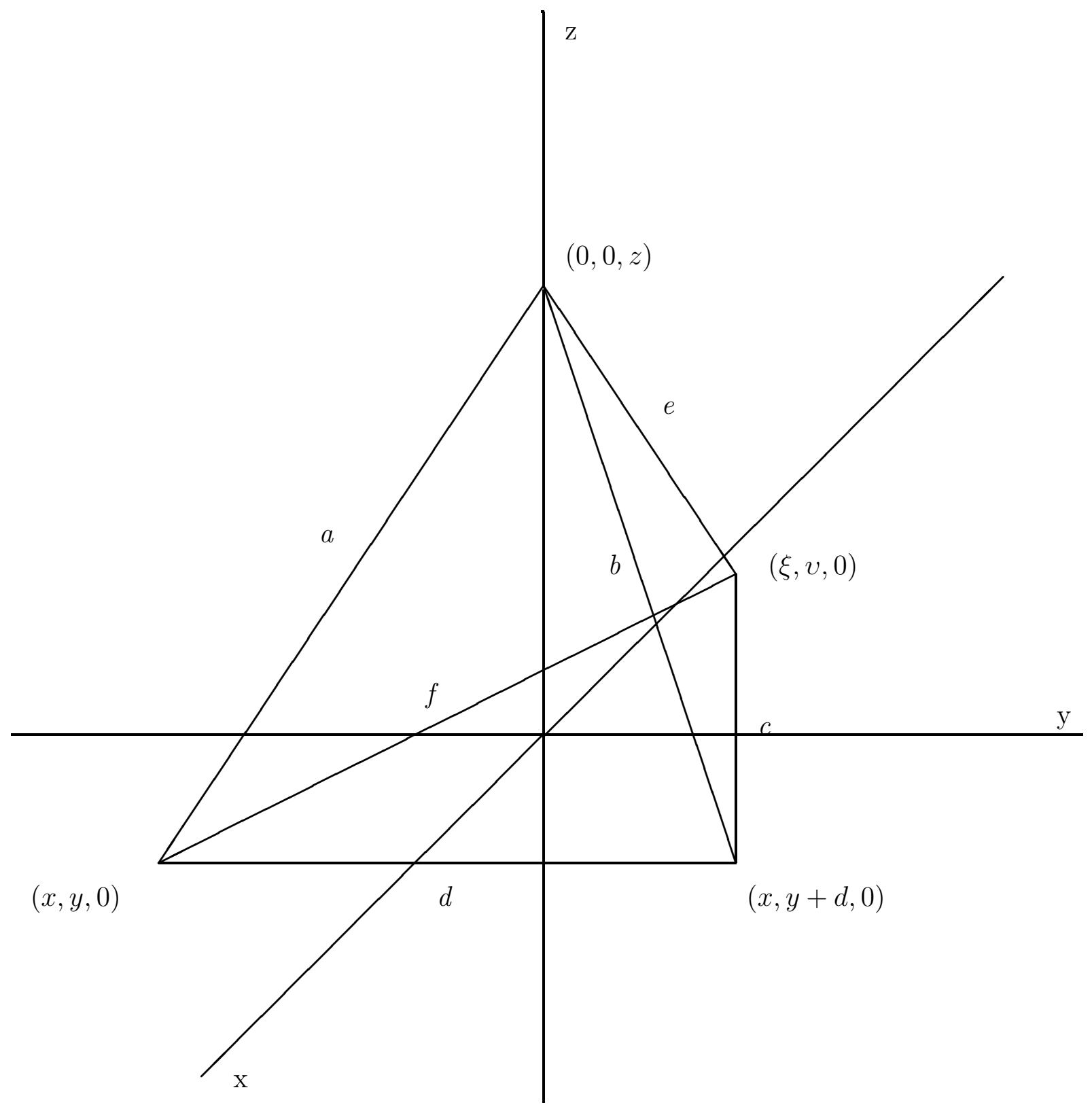

Figure 2: The tetrahedron coordinatized 
The medial tetrahedon is then spanned by the vectors $\vec{u}=\left(0, \frac{d}{2}, 0\right)$ and $\vec{v}=\left(-\frac{\xi}{2},-\frac{v}{2}, \frac{z}{2}\right)$, and thus

$$
\begin{aligned}
\text { Area } & =\|\vec{u} \times \vec{v}\| \\
& =\left\|\begin{array}{ccc}
\vec{i} & \vec{j} & \vec{k} \\
0 & \frac{d}{2} & 0 \\
-\frac{\xi}{2} & -\frac{v}{2} & \frac{z}{2}
\end{array}\right\| \\
& =\left\|\left(\frac{z d}{4}, 0, \frac{\xi d}{4}\right)\right\| \\
& =\frac{d}{4} \sqrt{z^{2}+\xi^{2}}
\end{aligned}
$$

Now consider the relationships between the coordinates and the edge lengths:

$$
\begin{aligned}
f^{2} & =(x-\xi)^{2}+(y-v)^{2} \\
c^{2} & =(x-\xi)^{2}+(y+d-v)^{2} \\
a^{2} & =x^{2}+y^{2}+z^{2} \\
b^{2} & =x^{2}+(y+d)^{2}+z^{2} \\
e^{2} & =\xi^{2}+v^{2}+z^{2}
\end{aligned}
$$

Subtracting Equation (7) from Equation (8), and solving the resulting equation for $y$ gives

$$
y=\frac{1}{2 d}\left(b^{2}-a^{2}-d^{2}\right) .
$$

Subtracting Equation (5) from Equation (6), substituting the value of $y$ from Equation (10), and solving for $v$ gives

$$
v=\frac{1}{2 d}\left(b^{2}+f^{2}-a^{2}-c^{2}\right)
$$

Now observe that by Equation (9) we have

$$
z^{2}+\xi^{2}=e^{2}-v^{2}
$$

Substituting this, and the value for $v$ from Equation (11) into Equation (4) and simplifying gives the desired result. $\square$. 


\section{References}

[1] Barbieri, A., "Quantum tetrahedra and simplicial spin networks," e-print gr-qc/9707010.

Barbieri, A., "Space of vertices of relativistic spin networks," e-print grqc/9709076.

[2] Barrett, J.W. and Crane, L., "Relativistic spin networks and quantum gravity," e-print gr-qc/9709028. 